\title{
Investigating the Biomarkers of the Sasang Constitution via Network Pharmacology Approach
}

\author{
Won-Yung Lee $\mathbb{D}^{1},{ }^{1}$ Choong-Yeol Lee $\mathbb{D}^{1},{ }^{1}$ Chang-Eop Kim $\mathbb{D}^{1},{ }^{1}$ and Ji-Hwan Kim $\mathbb{D}^{2}$ \\ ${ }^{1}$ Department of Physiology, College of Korean Medicine, Gachon University, Seongnam, Republic of Korea \\ ${ }^{2}$ Department of Sasang Constitutional Medicine, College of Korean Medicine, Gachon University, Seongnam, Republic of Korea
}

Correspondence should be addressed to Ji-Hwan Kim; jani77@gachon.ac.kr

Received 4 November 2020; Revised 22 March 2021; Accepted 3 April 2021; Published 14 April 2021

Academic Editor: Maria Grazia Ferraro

Copyright $\odot 2021$ Won-Yung Lee et al. This is an open access article distributed under the Creative Commons Attribution License, which permits unrestricted use, distribution, and reproduction in any medium, provided the original work is properly cited.

Sasang constitutional (SC) medicine classifies people into Soeum (SE), Soyang (SY), Taeeum (TE), and Taeyang (TY) types based on psychological and physical traits. However, biomarkers of these types are still unclear. We aimed to identify biomarkers among the SC types using network pharmacology methods. Target genes associated with the SC types were identified by grouping herb targets that preserve and strengthen the requisite energy (Bomyeongjiju). The herb targets were obtained by constructing an herb-compound-target network. We identified 371, 185, 146, and 89 target genes and their unique biological processes related to SE, SY, TE, and TY types, respectively. While the targets of SE and SY types were the most similar among the target pairs of the SC types, those of TY type overlapped with only a few other SC-type targets. Moreover, SE, SY, TE, and TY were related to "diseases of the digestive system," "diseases of the nervous system," "endocrine, nutritional, and metabolic diseases," and "congenital malformations, deformations, and chromosomal abnormalities," respectively. We successfully identified the target genes, biological processes, and diseases related to each SC type. We also demonstrated that a drug-centric approach using network pharmacology analysis provides a deeper understanding of the concept of Sasang constitutional medicine at a phenotypic level.

\section{Introduction}

The Sasang constitutional medicine (SCM) is a unique form of personalized medicine within traditional Korean medicine, which uses a constitutional typology for medical purposes. Unlike traditional Chinese medicine, which classifies the organs into five organ systems, the SCM theory classifies human physiological and pathological phenomena as four organ systems, which are paired: the lung, spleen, liver, and kidney. The liver-lung pair and spleen-kidney pair exhibit a functional symmetrical relationship similar to a seesaw. A hyperactive kidney and hypoactive spleen are traits unique to the Soeum (SE) type, and vice versa for the Soyang (SY) type. A hyperactive liver and hypoactive lung are applicable to the Taeeum (TE) type, and vice versa for the Taeyang (TY) type. The combination of their functional deviations represents the characteristics of the four Sasang constitutional (SC) types [1]. To identify those "inherent" characteristics, SCM experts categorize patients as one of the four SC types by comprehensively considering appearance, body shape, personality, and usual health status $[2,3]$. Using the diagnostic information of a patient's SC type, an SCM expert can identify the risk factors for certain SC-typespecific symptoms and provide a patient with tailored treatment. Several studies have reported that chronic diseases such as hypertension, obesity, and metabolic syndrome exhibit an SC-type-specific susceptibility [4-7]. Thus, although there may be controversy over the accuracy of the model, which classifies individual differences into four types according to the philosophical theory of a seesaw-like relationship, SCM can provide valuable experiences and helpful insights for improvement in personalized medicine, especially, in predicting prognosis and providing tailored interventions.

A prerequisite step for securing scientific evidence for SCM and expanding its application in personalized medicine is to identify biomarkers of each SC type. Previous studies, employing genome-wide association study (GWAS) and 
metabolomics study, have revealed genetic variations and metabolite-level biomarkers in healthy people [8-12]. However, the diagnosis of an SC type in these studies ultimately relied on the SCM doctors or questionnaires and was thus subject to bias due to the doctor's subjective clinical experience or low sensitivity of the questionnaire [13]. Therefore, there is still a pressing need for another approach that elucidates the biomarkers of SC types without bias.

On the basis of the SCM theory, we devised an alternative strategy to identify the biomarkers of the SC type. The energy (Qi) derived from the hypoactive organ is referred to as "the requisite energy (Bomyeongjiju, a life-sustaining source of power)" [14]. When this requisite energy is depleted, the equilibrium states of the visceral function of the SC types are disturbed and manifest as SC-type-specific symptoms or diseases. To alleviate such pathologies, corresponding medicinal herbs that preserve and strengthen the requisite energy for each SC type are prescribed. Consequently, the analysis of the mechanisms of medicinal herbs related to the requisite energy will help identifying the biomarkers associated with the inherent paired organs of each SC type and facilitate a deeper understanding of the SC types. We explored the mechanisms of these medicinal herbs using network pharmacology methods, which have emerged as promising tools for elucidating and analyzing the mechanisms of drugs at the system level [15-17]. The target genes of each SC type were identified by constructing the SCtype-related networks, and their related functions and diseases were analyzed and visualized (Figure 1). Given that these medicinal herbs are administered to treat a pathological condition specific to an SC type, the proposed approach, which we call it as "drug-centric approach," can provide clues for understanding biomarkers of the SC type at a phenotypic level.

\section{Materials and Methods}

2.1. Identification of Herbs. The list of herbs that strengthen and preserve the requisite energy (Bomyeongiiju) was obtained from Dongmuyugo, a classic Korean medical text written by Je-Ma Lee [18]. Among the books written by JeMa Lee, this is the only book that explicitly classifies medicinal herbs that enhance and preserve the energy needed for the SE, SY, and TE types. Another book written by Je-Ma Lee, Donguisusebowon (Longevity \& Life Preservation in Eastern Medicine), describes more abundant herbs that are prescribed for various symptoms and diseases of each SC type; nevertheless, it does not provide explicit links between herbs and the requisite energy [19]. However, even in Dongmuyugo, the herbs that conserve and strengthen the requisite energy for the TY type are not defined. Therefore, these herbs were inevitably defined as the common herbs in Ohgaphijangchuck-tang and Meehudeungsikjang-tang, the only two prescriptions for the TY type specified in Donguisusebowon [20]. The Latin names of herbs were retrieved from the KIOM herbarium (https://boncho.kiom.re.kr/ herbarium/codex.php) and the Korean Herbal Pharmacopoeia. The KIOM herbarium is a publicly accessible website that provides curated information, such as the origin and
Latin names of herbal medicines recorded in the Korean, Chinese, Japanese, Taiwanese, and North Korean pharmacopoeias. The above curation process was conducted after consultation with Korean doctors specialized in herbology or SCM.

2.2. Identification of Target Genes. The information of the target genes for each SC-type-related herb was obtained by constructing an herb-compound-target network using TCM-Mesh (http://mesh.tcm.microbioinformatics.org) [21]. TCM-Mesh is an online tool that provides a network pharmacology analysis of herbal medicines in a highthroughput manner by integrating information from various sources. For obtaining the information on compound-target interactions, the confidence for the interaction was set to the default value suggested by TCM-Mesh (combined score > 700). The score was calculated using a model that predicts the drug-target interactions based on a random forest model [22]. We limited the maximum number of target genes for a compound to 10 to avoid a biased result due to small sets of compounds with an immoderate number of target genes.

\subsection{Comparison and Visualization of Target Genes.} InteractiVenn (http://www.interactivenn.net) was employed to construct Venn diagrams between the target sets of the SC types [23]. This tool offers a clean interface for Venn diagram construction and enables the analysis of set unions while preserving the shape of the diagram. The target genes for each SC type were hierarchically clustered using the unweighted pair group method with arithmetic mean clustering algorithm with cosine distance.

2.4. Functional Annotation and Analysis. The functional annotation and gene ontology overrepresentation analyses were performed using the online analytical tool PANTHER (Protein ANalysis THrough Evolutionary Relationships; http://www.pantherdb.org, v.14.0) [24]. PANTHER is widely used as a comprehensive resource for gene function classification and genome-wide data analysis. Fisher's exact tests with the Benjamini-Hochberg false discovery rate correction were employed to determine the significance of gene ontology (GO) terms in the biological process category of the Homo sapiens genome. The $Z$-score of each GO term was computed for the deviation from an expected rank using a modification to Fisher's exact tests. Finally, the combined scores were calculated by multiplying the $Z$-scores and the logarithms of $p$ values (this combined score was notably different from the combined score in TCM-Mesh).

The involvement on diseases of each SC type was analyzed according to the procedure of a previously described method [25]. In brief, the gene-disease interactions were retrieved from the DisGeNET (https://www.disgenet.org/, v.6.0) [26]. The DisGeNET provides a score for manually curated gene-disease interactions between 0 and 1, taking into account the number of sources supporting the association and the reliability of each of them. The top three scored diseases associated with each target gene were 


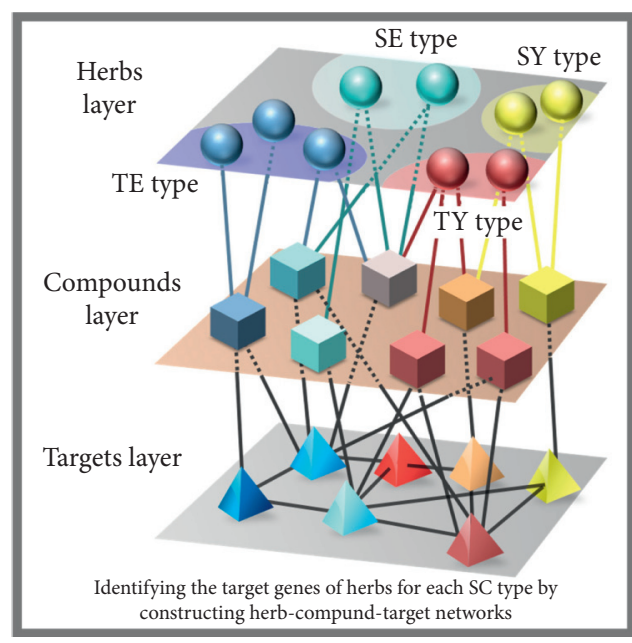

(a)

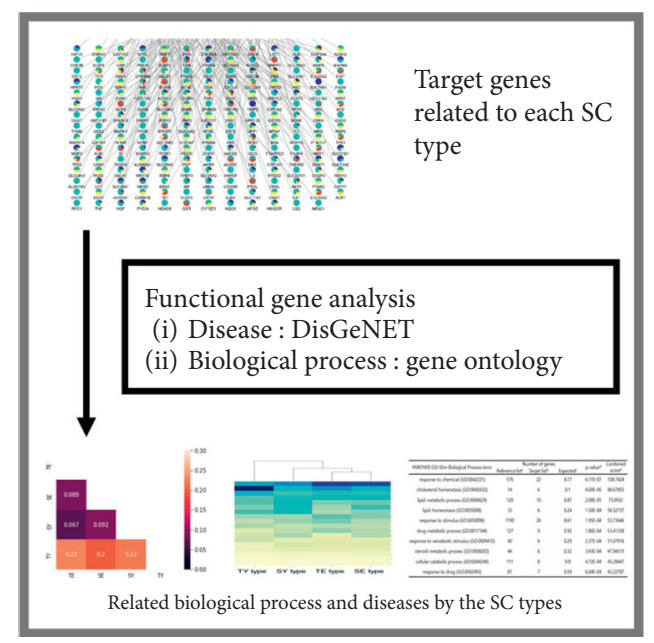

(b)

Figure 1: Overview of the study process. (a) Network construction. The SC-type-related networks were constructed by conducting a network pharmacology analysis on the herbs that preserve and strengthen the requisite energy in each SC type and integrating the obtained SC-type herb-compound-target interaction information. (b) Functional gene analysis. The functions and diseases for each SC type were identified by performing functional gene analyses on the target genes according to the SC type. SE: Soeum, SY: Soyang, TE: Taeeum, and TY: Taeyang.

obtained and classified based on the "International Statistical Classification of Diseases and Related Health Problems, 10th Revision (ICD-10): Version 2016” [27].

\section{Results}

3.1. Herb List Related to the Requisite Energy. To investigate the biological correlates of the SC types, we first identified the list of herbs that preserve and strengthen the requisite energy for each SC type. This list of herbs was retrieved from Dongmuyugo or defined using herbal formulae (see Materials and Methods). Among the herb list, Pruni Arillus (Aengdoyuk, the TY-type-related herb), which is not included in TCM-Mesh, was excluded from this study. Finally, a total of 28 herbs were included in the study (Table 1).

3.2. Target Genes Related to SC Type. To identify the target genes related to the SC types, we constructed an herbcompound-target network for each herb using TCM-Mesh (Figure 2 and Supplementary Table 1). The network consisted of three types of nodes (herbs, compounds, and targets) and two types of edges (between herbs and compounds and between compounds and targets). SC-type-related genes were retrieved by collecting those target genes of each SC type. We identified 371, 185, 146, and 89 related target genes of the SE, SY, TE, and TY types, respectively. We observed that the SE type and SY type shared the greatest number of target genes $(n=58)$ among the SC type pairs and so were grouped at the first level in the hierarchical clustering (Figure 3). This indicated that the target genes of the SE-SY pair were most similar among the target pairs of the SC type. On the other hand, the TY type shared only a few target genes with other SC types $(2,19$, and 0 for the TY-TE, TY-SE, and TY-SY pairs, respectively) and clustered at the last level, indicating that the target genes of the TY type were relatively distinct from those of other SC types.

Since we had limited the maximum number of target genes for a compound to 10, we tested whether this constraint biased the similarity relationship among the SC types. Therefore, we clustered the target genes related to the SC types by changing the threshold of the maximum target gene number $(n=10,50,100,500$, and no limit) and compared the order of clustering between them. The results showed that the hierarchy of clusters between the SC types did not change with varying thresholds, indicating that the similarity relationship among the SC types did not arise from the maximum number of target genes (Supplementary Figure 1).

Additionally, we tested whether the proportion of the target genes exclusively belonging to a specific SC type was higher than the values in the null distribution. The values of the null distribution were obtained by randomly shuffling the compound-target interactions and then repeatedly calculating the proportion of target genes belonging to a specific SC type. The results showed that the proportion of exclusive target genes in the original data was much higher than the chance level ( $p$ value $<0.001$ ), indicating that the group of target genes showed a unique pattern for each SC type.

3.3. Biological Correlates Related to Functional Gene Analysis. We annotated and analyzed the related functions of the target genes associated with the SC types. This analysis allows us to identify the biological processes associated with the target genes of each SC type. Every target gene of the compound-target network was assigned to first-level categories of biological processes using the "GO-Slim biological process" in the PANTHER classification system (v. 14.0) [24]. Among these categories, cellular processes, metabolic 
TABLE 1: List of herbs related to the requisite energy.

\begin{tabular}{|c|c|c|c|}
\hline No. & Sasang constitutional type & Local name & Latin name \\
\hline 1 & SE type & Insam (renshen) & Ginseng Radix \\
\hline 2 & & Baekchul (baizhu) & Atractylodis Rhizoma Alba \\
\hline 3 & & Jagamcho (zhigancao) & Glycyrrhizae Radix et Rhizoma Praeparata cum Melle \\
\hline 4 & & Danggui (danggui) & Angelicae Gigantis Radix \\
\hline 5 & & Chungung (chuanxiong) & Cnidii Rhizoma \\
\hline 6 & & Gwangye (guangui) & Cortex Cinnamomi \\
\hline 7 & & Jinpi (chenpi) & Citri Unshius Pericarpium \\
\hline 8 & & Baekjakyak (baishaoyao) & Paeoniae Radix \\
\hline 9 & SY type & Sugjihwang (shudihuang) & Rehmanniae Radix Preparata \\
\hline 10 & & Sansuyu (shanzhuyu) & Corni Fructus \\
\hline 11 & & Bokryeong (fufing) & Poria Sclerotium \\
\hline 12 & & Jimo (zhimu) & Anemarrhenae Rhizoma \\
\hline 13 & & Taegsa (zexie) & Alismatis Rhizoma \\
\hline 14 & & Moktong (mutong) & Akebiae Caulis \\
\hline 15 & & Mokdanpi (mudanpi) & Moutan Radicis Cortex \\
\hline 16 & & Huangbaek (huangbai) & Phellodendri Cortex \\
\hline 17 & TE type & Maengmundong (maimendong) & Liriopis seu Ophiopogonis Tuber \\
\hline 18 & & Omija (wuweizi) & Schisandrae Fructus \\
\hline 19 & & Sadang (shatang) & Saccharum \\
\hline 20 & & Sanyak (shanyao) & Dioscoreae Rhizoma \\
\hline 21 & & Gilgyeong (jiegeng) & Platycodonis Radix \\
\hline 22 & & Uwang (niuhuang) & Bostaurus Linne var. domesticus Gmelin \\
\hline 23 & & Seokchangpo (shichangpu) & Acori Graminei Rhizoma \\
\hline 24 & & Hunaggeum (huangqin) & Scutellariae Radix \\
\hline 25 & TY type ${ }^{\#}$ & Ogapi (wejiapi) & Acanthopanacis Cortex \\
\hline 26 & & Mogwa (mugua) & Chaenomelis Fructus \\
\hline 27 & & Podogeun (putaogen) & Radix Vitis Romanetii \\
\hline 28 & & Nogeun (lugen) & Phragmitis Rhizoma \\
\hline
\end{tabular}

SE: Soeum, SY: Soyang, TE: Taeeum, and TY: Taeyang. \# The herbs for the TY type were selected from herbal formulae prescribed for the TY type (see Materials and Methods for detail).

processes, and biological regulation were highly associated with the target genes of the SC types (Supplementary Table 2). In particular, more than half of the target genes of the TY type were found to be involved in metabolic processes. Simultaneously, we also calculated the Euclidean distance for the proportion of the categories of biological processes for each SC-type pair (Figure 4). The SY-TE pair presented the lowest distance among all constitution pairs, followed by SE-TE and SE-SY pairs (0.067, 0.089, and 0.092, respectively). On the other hand, all TY-type-related pairs showed the greatest distance from the other pairings $(0.2,0.22$, and 0.23 for TY-SE, TY-SY, and TY-TE pairs, respectively), thus indicating that the function of the TY type was very different from the other SC types.

Next, we conducted an overrepresentation test to investigate the specific biological processes significantly associated with each SC type using PANTHER (v.14.0). The results showed that $74,86,43$, and 60 biological process terms were significantly associated to the SE, SY, TE, and TY types, respectively (adjusted $p<0.05$, Tables 2-5, and Supplementary Tables 3-6). Among the top 10 terms, ranked by the combined score, the drug metabolic process (GO: 0017144) and response to chemical (GO:0042221) terms were commonly associated with the SE, SY, and TE types. On the other hand, each SC type showed association with distinct terms in the biological process category. The SE type was significantly related to various metabolic processes (GO:
0008152) (cellular metabolic process, fatty acid metabolic process, cofactor metabolic process, carbohydrate metabolic process, cellular lipid metabolic process, ammonium ion metabolic process, nitrogen compound metabolic process; Table 3), whereas the SY type was significantly associated with the positive regulation of lymphocyte proliferation (GO:0050671), leukocyte proliferation (GO:0070665), and mononuclear cell proliferation (GO:0032946) (Table 4). While the TE type was significantly associated with lipid homeostasis (GO:0006629)-related and lipid metabolism (GO:0055088)-related terms (cholesterol homeostasis, lipid metabolic process, lipid homeostasis, steroid metabolic process; Table 2), the TY type was significantly related to ATP-associated metabolic processes (GO:0046034) (mitochondrial electron transport, cytochrome $\mathrm{c}$ to oxygen, and ATP metabolic process; Table 5).

To characterize the diseases in each SC type, we analyzed and compared the association of the three top-scoring disease categories with each SC type using DisGeNET (Figure 5). The "neoplasms" showed a high frequency in most of the groups, a result consistent with that of the previous research that identified diseases related to medical herbs [25]. On the other hand, each SC type showed differences in the annotated disease classifications; "endocrine, nutritional, and metabolic diseases" showed the highest frequency $(20.0 \%)$ in the TE type, and "diseases of the digestive system" showed the third highest frequency $(9.4 \%)$ in 


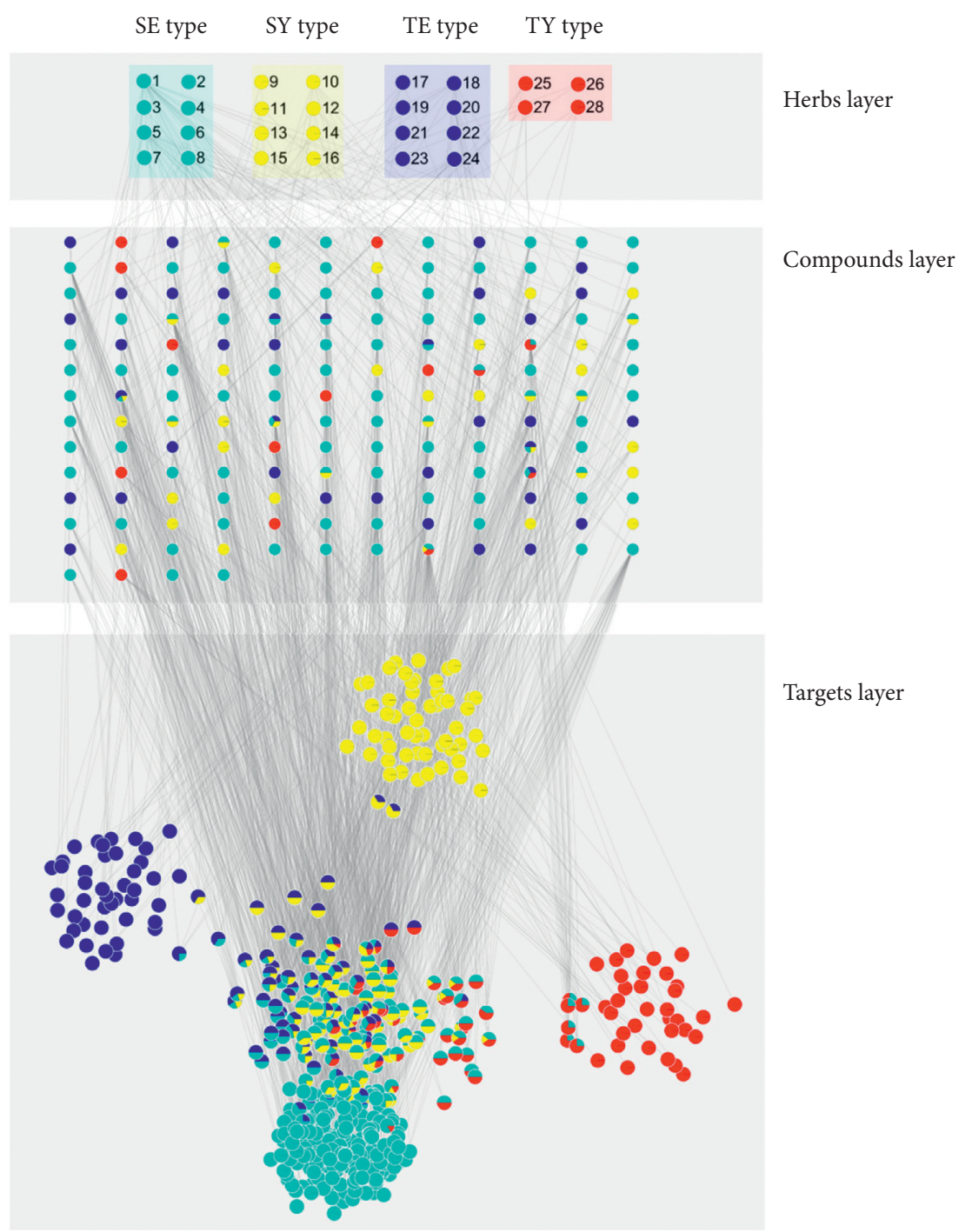

FiguRE 2: Representative herb-compound-target networks for herbs related to the requisite energy. The circles in each layer represent herbs, compounds, or targets. The pie charts in each node denote proportions related to the SC types (Blue, cyan, yellow, and red colors represent TE, SE, SY, and TY types, respectively.). The herb no. (see Table 1) and gene symbols are attached to the nodes in the herbs layer and targets layer. SE: Soeum, SY: Soyang, TE: Taeeum, and TY: Taeyang.

the SE type. "Diseases of the nervous system" and "mental, behavioral, and neurodevelopmental disorders" showed the second and seventh highest prevalence, respectively $(9.8 \%$ and $7.8 \%$ ), in the SY type, and "congenital malformations, deformations, and chromosomal abnormalities" showed the third highest frequency $(11.3 \%)$ in the TY type.

\section{Discussion}

Through this study, we have addressed a crucial issue in SCM by demonstrating that a drug-centric approach can successfully uncover novel biomarkers for each SC type with related biological functions and diseases. The drug-centric approach often refers to a drug discovery strategy that links known drugs to new targets and their related indications [28]. However, in this study, it refers to the alternative strategy of identifying biomarkers associated with the SC type, as established by the SCM theory with inherent paired organs and the requisite energy, by using the modern approach of network pharmacology for analyzing medicinal herbs that are classified according to the same SCM theory. The permutation test supports that the group of target genes between SC types is clearly distinguished compared with the random distribution, and this unique pattern is reflected in the results for biological processes and diseases.

It is noteworthy that our results of the SC-type-related genes differ from those of previous studies, which addressed the characteristics of genetic background for each SC type. Kim et al. found that polymorphisms of some genes, such as FTO, MC4R, and interleukin- $1 \alpha$ and $\beta$, were associated with SC type [8-10], which was not corroborated by our results. Such inconsistency may have been derived from the research hypotheses and the employed experimental techniques. Above-mentioned previous studies have identified genetic variations using GWAS based on the hypothesis that there are inherited characteristics 


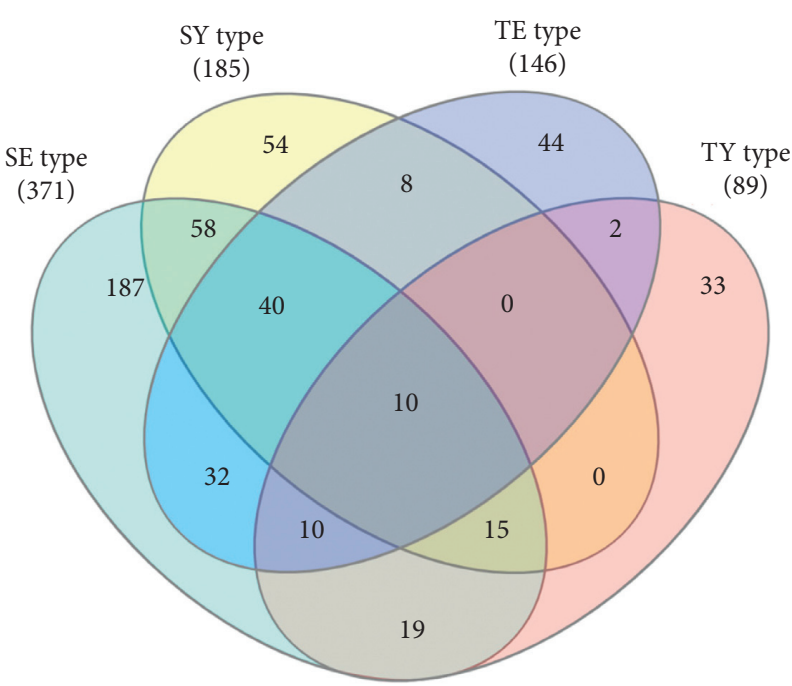

(a)

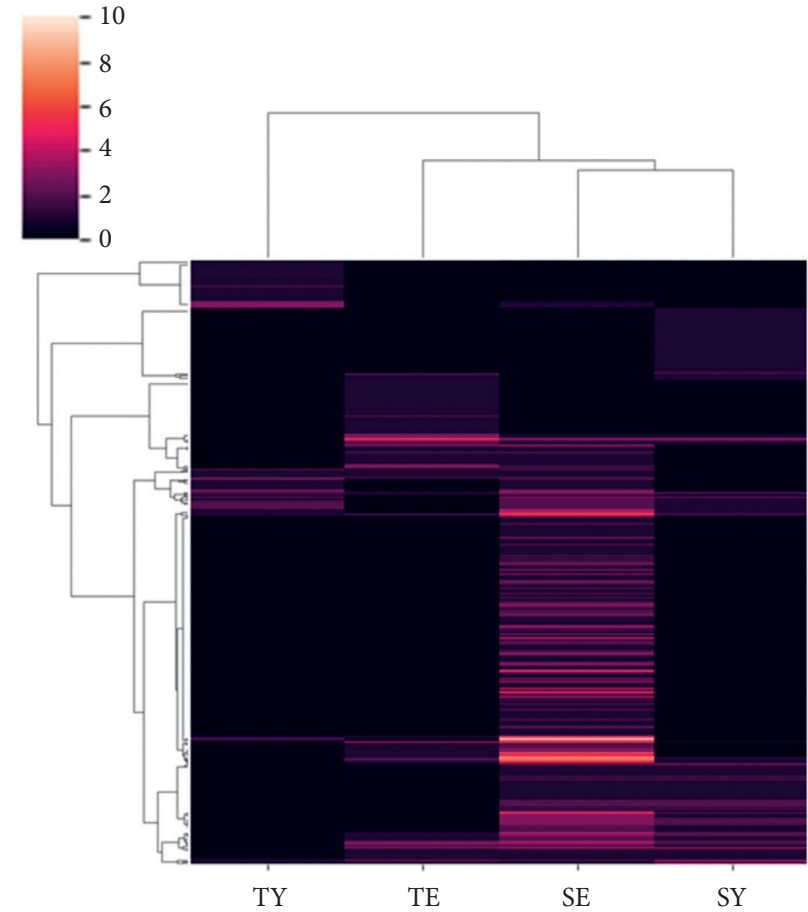

(b)

(a)

(b)

FIGURE 3: Comparison among the related targets of Sasang constitutional (SC) types. (a) Venn diagram of the targets related to SC type. The number indicates the targets that were shared between or unique to each SC type. (b) Clustered heatmap of the targets related to SC types. Each line represents a specific target, and the color of the line indicates the number of herbal compounds interacting with each target. The data are displayed in a heatmap and clustered with respect to both targets and SC types. SE: Soeum, SY: Soyang, TE: Taeeum, and TY: Taeyang.

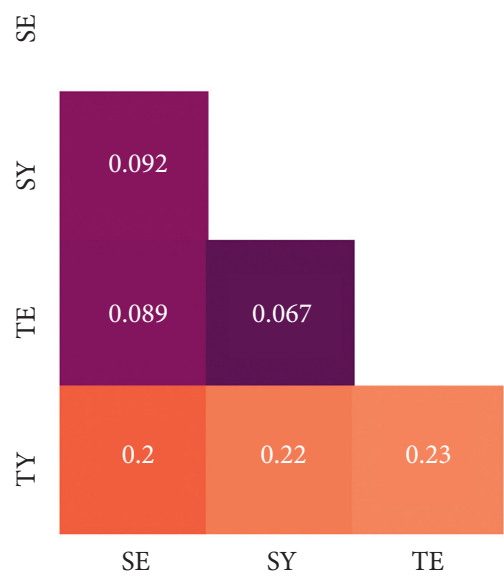

Figure 4: Distance matrix of the SC types. The color bar indicates the Euclidian distance between the SC types in terms of their related biological processes. SE: Soeum, SY: Soyang, TE: Taeeum, and TY: Taeyang.

associated with specific genes that are related to phenotypic traits of each SC type. On the other hand, we focused on the targets of herbs and their associated biological processes and diseases based on the hypothesis that medicinal herbs prescribed to patients are the key to understanding the pathophysiological traits of the SC type [18]. Since SCM is actually based on the clinical practice using specific herbs for each SC type, our drug-centric approach can provide a further explanation about the pathophysiological traits of the SC types in the clinical setting.

Our cluster analysis revealed that the target genes between the SE type and SY type were most similar among the target gene list of the SC-type pairs (Figure 3). This result may seem controversial because the SE and SY types exhibit opposite activities with respect to the kidney and spleen groups [1]. One possible explanation for this contradictory result is that the medicinal herbs assigned to the SE and SY types act on the overlapped target gene through the opposite mode of action. Since current network pharmacology methods only predict simple interactions without the mode of action, further research is needed for identifying biomarkers of the SC type that exhibit upregulation and downregulation. However, our results on the Euclidian distance of the related biological process among the SC types indicated that the SY type and TE type were the closest pair in the biological process category. The two types share more similarity than the SE or TY type in that they show a cold pattern in exterior disease and a heat pattern in internal disease, while SE shows the reverse pattern, and TY does not show a distinct heat or cold pattern [20]. Our results 
TABLE 2: Gene ontology terms of the biological process category associated with Soeum type.

\begin{tabular}{|c|c|c|c|c|c|}
\hline \multirow{2}{*}{ PANTHER GO-Slim biological process term } & \multicolumn{3}{|c|}{ Number of genes } & \multirow{2}{*}{$p$ value $^{\mathrm{d}}$} & \multirow{2}{*}{ Combined score } \\
\hline & Reference list ${ }^{\mathrm{a}}$ & Target list ${ }^{\mathrm{b}}$ & Expected $^{\mathrm{c}}$ & & \\
\hline Response to xenobiotic stimulus (GO:0009410) & 40 & 12 & 0.37 & $5.19 E-12$ & 205.5949 \\
\hline Drug metabolic process (GO:0017144) & 127 & 21 & 1.19 & $5.60 E-12$ & 342.0589 \\
\hline Response to drug (GO:0042493) & 81 & 16 & 0.76 & $8.09 E-09$ & 252.1907 \\
\hline Terpenoid metabolic process (GO:0006721) & 11 & 5 & 0.1 & $9.17 E-09$ & 63.41361 \\
\hline Isoprenoid metabolic process (GO:0006720) & 14 & 5 & 0.13 & $4.93 E-08$ & 57.00586 \\
\hline Diterpenoid metabolic process (GO:0016101) & 10 & 4 & 0.09 & $2.56 E-07$ & 41.92858 \\
\hline Positive regulation of leukocyte proliferation (GO:0070665) & 3 & 2 & 0.03 & $3.39 E-07$ & 16.30587 \\
\hline Regulation of smooth muscle contraction (GO:0006940) & 3 & 2 & 0.03 & $3.56 E-07$ & 16.26849 \\
\hline $\begin{array}{l}\text { Positive regulation of mononuclear cell proliferation (GO: } \\
\text { 0032946) }\end{array}$ & 3 & 2 & 0.03 & $1.14 E-06$ & 16.21936 \\
\hline Positive regulation of lymphocyte proliferation (GO:0050671) & 3 & 2 & 0.03 & $2.53 E-06$ & 16.18301 \\
\hline
\end{tabular}

${ }^{a}$ Number of genes in the reference list that map to this PANTHER classification category. ${ }^{b}$ Number of genes in the target gene list that map to this PANTHER classification category. ${ }^{c}$ The expected value is the number of genes that can be expected in the target gene list for this PANTHER category based on the reference list. ${ }^{\mathrm{d}} p$ values are determined by binomial statistics with Benjamini-Hochberg corrections. ${ }^{\mathrm{e}}$ Combined score is computed by taking the log of the $p$ value and multiplying that by the $Z$-score of the deviation from the expected rank.

TABLE 3: Gene ontology terms of the biological process category associated with Soyang type.

\begin{tabular}{|c|c|c|c|c|c|}
\hline \multirow{2}{*}{ PANTHER GO-Slim biological process term } & \multicolumn{3}{|c|}{ Number of genes } & \multirow{2}{*}{$p$ value $^{\mathrm{d}}$} & \multirow{2}{*}{ Combined score } \\
\hline & Reference list ${ }^{\mathrm{a}}$ & Target list ${ }^{\mathrm{b}}$ & Expected $^{\mathrm{c}}$ & & \\
\hline Cellular metabolic process (GO:0044237) & 1744 & 84 & 32.73 & $7.02 E-16$ & 230.1149 \\
\hline Drug metabolic process (GO:0017144) & 127 & 23 & 2.38 & $5.71 E-13$ & 226.2333 \\
\hline Fatty acid metabolic process (GO:0006631) & 138 & 20 & 2.59 & $2.50 E-11$ & 144.3277 \\
\hline Response to chemical (GO:0042221) & 576 & 39 & 10.81 & $9.88 E-10$ & 141.426 \\
\hline Response to xenobiotic stimulus (GO:0009410) & 40 & 12 & 0.75 & $4.88 E-08$ & 123.8619 \\
\hline Cofactor metabolic process (GO:0051186) & 159 & 19 & 2.98 & $1.32 E-07$ & 107.5996 \\
\hline Cellular lipid metabolic process (GO:0044255) & 294 & 25 & 5.52 & $3.47 E-07$ & 103.8276 \\
\hline Carbohydrate metabolic process (GO:0005975) & 185 & 20 & 3.47 & $5.99 E-07$ & 103.5943 \\
\hline Ammonium ion metabolic process (GO:0097164) & 34 & 10 & 0.64 & $1.28 E-06$ & 92.36633 \\
\hline Nitrogen compound metabolic process (GO:0006807) & 38 & 10 & 0.71 & $1.41 E-06$ & 85.06531 \\
\hline
\end{tabular}

${ }^{a}$ Number of genes in the reference list that map to this PANTHER classification category. ${ }^{b}$ Number of genes in the target gene list that map to this PANTHER classification category. ${ }^{c}$ The expected value is the number of genes that can be expected in the target gene list for this PANTHER category based on the reference list. ${ }^{\mathrm{d}} p$ values are determined by binomial statistics with Benjamini-Hochberg corrections. ${ }^{\mathrm{e}}$ Combined score is computed by taking the log of the $p$ value and multiplying that by the $Z$-score of the deviation from the expected rank.

TABLE 4: Gene ontology terms of the biological process category associated with Taeeum type.

\begin{tabular}{|c|c|c|c|c|c|}
\hline \multirow{2}{*}{ PANTHER GO-Slim biological process term } & \multicolumn{3}{|c|}{ Number of genes } & \multirow[t]{2}{*}{ p-value ${ }^{\mathrm{d}}$} & \multirow[t]{2}{*}{ Combined score } \\
\hline & Reference list ${ }^{\mathrm{a}}$ & Target list $\mathrm{t}^{\mathrm{b}}$ & Expected $^{\mathrm{c}}$ & & \\
\hline Response to chemical (GO:0042221) & 576 & 22 & 4.17 & $6.11 E-07$ & 108.7424 \\
\hline Cholesterol homeostasis (GO:0042632) & 14 & 6 & 0.1 & $4.00 E-06$ & 88.67855 \\
\hline Lipid metabolic process (GO:0006629) & 120 & 10 & 0.87 & $2.08 E-05$ & 73.0932 \\
\hline Lipid homeostasis (GO:0055088) & 33 & 6 & 0.24 & $1.50 E-04$ & 56.52157 \\
\hline Response to stimulus (GO:0050896) & 1190 & 26 & 8.61 & $1.95 E-04$ & 53.73646 \\
\hline Drug metabolic process (GO:0017144) & 127 & 9 & 0.92 & $1.86 E-04$ & 53.41338 \\
\hline Response to xenobiotic stimulus (GO:0009410) & 40 & 6 & 0.29 & $2.37 E-04$ & 51.07916 \\
\hline Steroid metabolic process (GO:0008202) & 44 & 6 & 0.32 & $3.43 E-04$ & 47.94313 \\
\hline Cellular catabolic process (GO:0044248) & 111 & 8 & 0.8 & $4.72 E-04$ & 45.28447 \\
\hline Response to drug (GO:0042493) & 81 & 7 & 0.59 & $6.04 E-04$ & 43.22787 \\
\hline
\end{tabular}

${ }^{\mathrm{a}}$ Number of genes in the reference list that map to this PANTHER classification category. ${ }^{\mathrm{b}}$ Number of genes in the target gene list that map to this PANTHER classification category. ${ }^{c}$ The expected value is the number of genes that can be expected in the target gene list for this PANTHER category based on the reference list. ${ }^{\mathrm{d}} p$ values are determined by binomial statistics with Benjamini-Hochberg corrections. ${ }^{\mathrm{e}}$ Combined score is computed by taking the log of the $p$ value and multiplying that by the $\mathrm{Z}$-score of the deviation from the expected rank.

additionally showed that the biological processes, as well as target genes, of the TY type are different from those of the other SC types. Further research on this aspect is needed.
The functional gene analysis revealed the related biological process or diseases for each SC type. Although the underlying mechanisms of the SC-type-specific diseases 
TABLE 5: Gene ontology terms of the biological process category associated with Taeyang type.

\begin{tabular}{|c|c|c|c|c|c|}
\hline \multirow[b]{2}{*}{ PANTHER GO-Slim biological process term } & \multicolumn{3}{|c|}{ Number of genes } & \multirow[b]{2}{*}{$p$ value $^{\mathrm{d}}$} & \multirow[b]{2}{*}{$\begin{array}{c}\text { Combined } \\
\text { score }^{e}\end{array}$} \\
\hline & $\begin{array}{c}\text { Reference } \\
\text { list }^{\mathrm{a}}\end{array}$ & $\begin{array}{c}\text { Target } \\
\text { list }\end{array}$ & Expected $^{\mathrm{c}}$ & & \\
\hline Inorganic anion transport (GO:0015698) & 44 & 9 & 0.19 & $2.13 E-09$ & 164.4011 \\
\hline $\begin{array}{l}\text { Mitochondrial electron transport, cytochrome } \mathrm{c} \text { to oxygen (GO: } \\
0006123 \text { ) }\end{array}$ & 185 & 13 & 0.79 & $2.33 E-09$ & 159.3545 \\
\hline Nitric oxide biosynthetic process (GO:0006809) & 54 & 9 & 0.23 & $3.18 E-09$ & 152.3056 \\
\hline Sensory perception of sweet taste (GO:0050916) & 55 & 9 & 0.24 & $3.66 E-09$ & 152.0322 \\
\hline Arginine catabolic process (GO:0006527) & 66 & 9 & 0.28 & $1.01 E-08$ & 138.1824 \\
\hline Negative regulation of blood pressure (GO:0045776) & 10 & 6 & 0.04 & $1.11 E-08$ & 137.2376 \\
\hline Regulation of lyase activity (GO:0051339) & 42 & 8 & 0.18 & $1.12 E-08$ & 137.1961 \\
\hline Positive regulation of phosphate metabolic process (GO:0045937) & 45 & 8 & 0.19 & $1.21 E-08$ & 134.0532 \\
\hline Secondary metabolite biosynthetic process (GO:0044550) & 45 & 8 & 0.19 & $1.36 E-08$ & 133.5836 \\
\hline ATP synthesis coupled electron transport (GO:0042773) & 4072 & 45 & 17.45 & $1.48 E-08$ & 131.5255 \\
\hline
\end{tabular}

${ }^{a}$ Number of genes in the reference list that map to this PANTHER classification category. ${ }^{b}$ Number of genes in the target gene list that map to this PANTHER classification category. ${ }^{c}$ The expected value is the number of genes that can be expected in the target gene list for this PANTHER category based on the reference list. ${ }^{\mathrm{d}} p$ values are determined by binomial statistics with Benjamini-Hochberg corrections. ${ }^{\mathrm{e}} \mathrm{Combined}$ score is computed by taking the log of the $p$ value and multiplying that by the $Z$-score of the deviation from the expected rank.

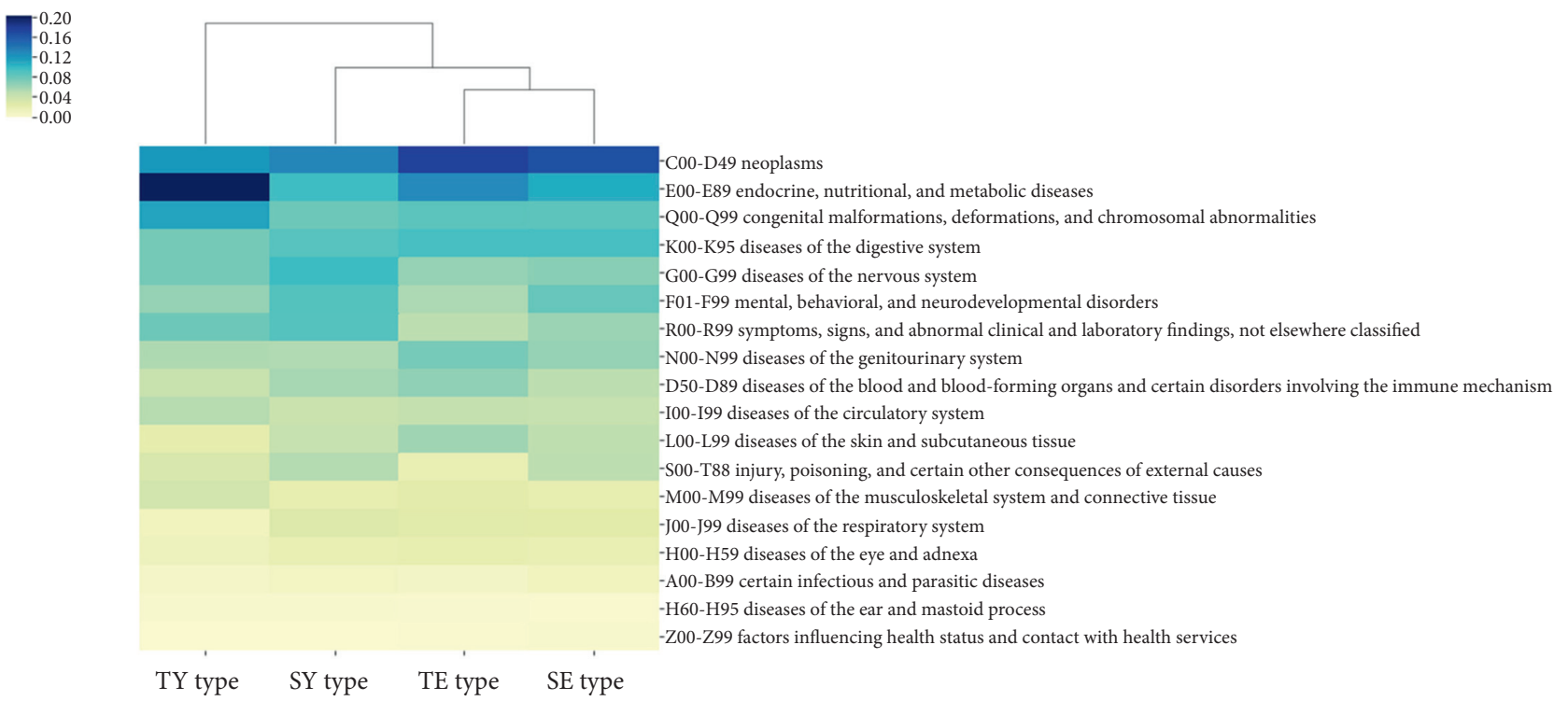

FIGURE 5: Related disease categories by Sasang constitutional (SC) type. The color of each cell denotes the proportion of diseases associated with each SC type. SE: Soeum, SY: Soyang, TE: Taeeum, and TY: Taeyang.

were not completely revealed, the results from the analysis provide clues to identify the underlying pathophysiology of the diseases for each SC type. For example, our results showed that the TE type is closely related to lipid homeostasis-related and lipid metabolism-related biological process terms and also highly related to the "endocrine, nutritional, and metabolic disease." Indeed, recent studies have shown that lipid homeostasis and lipid metabolism are closely associated with obesity, diabetes, and metabolic syndrome [29-31], and the prevalence of these diseases was found to be highest in the TE type $[5,32,33]$. Our results also identified that the SE type was closely associated with metabolic processes and the "disease of the digestive system." Similarly, recent researches revealed that the irritable bowel syndrome, a disease highly prevalent in the SE type [4], is positively related to the expression of genes involved in metabolic processes [34]. These consistent results prove that the techniques employed in our study are reliable and can be used to generate new hypotheses for future studies. Specifically, our results can help reveal the pathophysiological traits of the SY and the TY types, which have not yet been studied extensively.

One major advantage of the drug-centric approach is that the SC types cannot be falsely labeled. In the previous studies [8-12], the SC types were classified by the SCM doctors, after considering the patient's morphological and psychological traits. However, its diagnostic procedure can be biased by the doctor's subjective clinical experience, which means that the relative importance of traits contributing to SC-type diagnosis can vary from doctor to doctor. This situation often leads to the diagnosis of different SC types for the same person. One study reported that the 
accordance rates of SC-type diagnosis between SCM experts were 52.5-68.4\%, according to SC types [35]. Another advantage is that the drug-centric approach can identify biomarkers of rare constitutions that may be difficult to study in a population. For instance, the Korea Constitutional Multicenter Bank (KCMB), the largest biobank of SCM, revealed that the constitutional distributions for TY type were nonzero but below $0.1 \%$, deeming it too rare to include in a population-based study [36]. To the best of our knowledge, we first identified biomarkers of the TY type, leveraging prior knowledge from the classic Korean medical text.

There are some limitations to our study as well. First, our results are based on the hypothesis of Je-Ma Lee, so our findings require additional validation using preclinical and clinical studies. Second, our results cannot determine whether the target genes or biological functions are activated or inhibited in each SC type. An interesting objective for future research will be identifying which target genes or biological processes are upregulated or downregulated, to shed more light on the seesaw balance between the organ groups of specific pairs. Third, our scope of analysis has focused on the herbs that are explicitly related to the requisite energy, except certain frequently prescribed medicinal herbs such as Aconiti Lateralis, Radix Preparata, Gypsum Fibrosum, and Puerariae Radix for the SE, SY, and TY types, respectively. In spite of these limitations, our findings provide a deeper understanding of the concept of SCM at the biological level.

\section{Conclusion}

In this study, we identified biomarkers of SC type by employing network pharmacology. The target genes, biological processes, and diseases showed unique patterns among the SC types, and they were consistent with the results of previous studies. Furthermore, we showed that the proposed strategy can provide reliable hypothesis for SCM, which broadens the application of personalized medicine by bridging the gap between SCM and modern medicine.

\section{Data Availability}

The data used to support the findings of this study are included within the supplementary information files.

\section{Disclosure}

The funder has no role/influence in this study.

\section{Conflicts of Interest}

The authors declare that they have no conflicts of interest.

\section{Authors' Contributions}

JHK and CEK conceptualized the work. Data curation was carried out by WYL. Formal analysis was performed by WYL and CEK. Data visualization was carried out by WYL. Original draft was prepared by WYL. CYL, CEK, and JHK reviewed and edited the original draft to provide the final manuscript.

\section{Acknowledgments}

This work was supported by the National Research Foundation of Korea (NRF) grant funded by the Korea Government (MSIT) (NRF-2020R1F1A1065731).

\section{Supplementary Materials}

Figure S1: cluster map of the targets associated with Sasang constitution types by changing the threshold of the number of targets ( $n=10,50,100,500$, and no limit) of a compound. Supplementary Table 1: target genes related to each Sasang constitutional type. Supplementary Table 2: biological processes related to the targets of each Sasang constitutional type. Supplementary Table 3: gene ontology terms of the biological process category associated with Soeumin type. Supplementary Table 4: gene ontology terms of the biological process category associated with Soyangin type. Supplementary Table 5: gene ontology terms of the biological process category associated with Taeeumin type. Supplementary Table 6: gene ontology terms of the biological process category associated with Taeyangin type. (Supplementary Materials)

\section{References}

[1] J. Y. Kim and D. D. Pham, "Sasang constitutional medicine as a holistic tailored medicine," Evidence-Based Complementary and Alternative Medicine, vol. 6, Article ID 176507, 9 pages, 2009.

[2] S. W. Lee, E. S. Jang, J. Lee, and J. Y. Kim, “Current researches on the methods of diagnosing sasang constitution: an overview," Evidence-Based Complementary and Alternative Medicine, vol. 6, Article ID 390301, 7 pages, 2009.

[3] S.-Y. Park, M. Park, W.-Y. Lee et al., "Machine learning-based prediction of Sasang constitution types using comprehensive clinical information and identification of key features for diagnosis," Integrated Medicine Research, vol. 10, 2020.

[4] H.-Y. Lee, W.-J. Lee, H.-W. Kim et al., "A systematic review on Sasang constitutional type-associated susceptibility to disorders in Korea," The Journal of Alternative and Complementary Medicine, vol. 22, no. 12, pp. 950-956, 2016.

[5] E. Jang, Y. Baek, K. Park, and S. Lee, "Could the Sasang constitution itself be a risk factor of abdominal obesity?" BMC Complementary and Alternative Medicine, vol. 13, no. 72, pp. 1-6, 2013.

[6] S. K. Lee, D. W. Yoon, S. W. Lee et al., "Non-alcoholic fatty liver disease among sasang constitutional types: a populationbased study in Korea," BMC Complementary and Alternative Medicine, vol. 15, no. 399, pp. 1-7, 2015.

[7] K. H. Song, S. G. Yu, and J. Y. Kim, "Prevalence of metabolic syndrome according to sasang constitutional medicine in Korean subjects," Evidence-Based Complementary and Alternative Medicine, vol. 2012, Article ID 646794, 8 pages, 2012.

[8] J. Y. Kim, S. Cha, I. Koo et al., "Genetic effects of FTO and MC4R polymorphisms on body mass in constitutional types," Evidence-Based Complementary and Alternative Medicine, vol. 2011, Article ID 106390, 7 pages, 2011.

[9] J.-H. Lee, Y.-D. Kwon, S.-H. Hong, H.-J. Jeong, H.-M. Kim, and J.-Y. Um, "Interleukin-1 beta gene polymorphism and traditional constitution in obese women," International Journal of Neuroscience, vol. 118, no. 6, pp. 793-805, 2008. 
[10] B.-Y. Kim, S.-G. Yu, J.-Y. Kim, and K. H. Song, "Pathways involved in sasang constitution from genome-wide analysis in a Korean population," The Journal of Alternative and Complementary Medicine, vol. 18, no. 11, pp. 1070-1080, 2012.

[11] M. J. Kim, D. H. Lee, J. Ahn et al., "A pilot study on characteristics of metabolomics and lipidomics according to sasang constitution," Evidence-Based Complementary and Alternative Medicine, vol. 2018, Article ID 9214960, 12 pages, 2018.

[12] E. J. Kim, Y. S. Hong, S. H. Seo et al., "Metabolite markers for characterizing sasang constitution type through GC-MS and 1 H NMR-based metabolomics study," Evidence-Based Complementary and Alternative Medicine, vol. 2019, Article ID 8783496, 11 pages, 2019.

[13] S. H. Kim, Y. Lee, B. H. Koh, and E. Jang, "Assessing the diagnostic accuracy of the questionnaire for sasang constitutional classification II (QSCC II): a systematic review," European Journal of Integrative Medicine, vol. 5, 2013.

[14] B. H. Koh, J. Y. Kim, and D. D. Pham, "Comparison of sasang constitutional medicine, traditional Chinese medicine and ayurveda," Evidence-Based Complementary and Alternative Medicine, vol. 2011, Article ID 239659, 6 pages, 2011.

[15] W.-Y. Lee, C.-Y. Lee, Y.-S. Kim, and C.-E. Kim, "The methodological trends of traditional herbal medicine employing network pharmacology," Biomolecules, vol. 9, no. 8 , p. 362, 2019.

[16] S.-Y. Park, J.-H. Park, H.-S. Kim et al., "Systems-level mechanisms of action of Panax ginseng: a network pharmacological approach," Journal of Ginseng Research, vol. 42, no. 1, pp. 98-106, 2018.

[17] D. H. Lee, W. Y. Lee, K. W. Jung et al., "The inhibitory effect of cordycepin on the proliferation of MCF-7 breast cancer cells, and its mechanism: an investigation using network pharmacology-based analysis," Biomolecules, vol. 9, no. 9, p. 407, 2019.

[18] D. P. Won, Gugyeokanuihakdaegye 15-Dong-Mu-Yu-Go, Haedonguihaksa, Seoul, Korea, 1st edition, 1999.

[19] P. Seong-Sik, "The study on the DongMuYooGo YakSungGa," Journal of Sasang Constitutional Medicine, vol. 13, no. 2, pp. 8-27, 2001.

[20] Department of Sasang Constitutional Medicine, Colleges of Korean Medicine in Korea (Compilation), the Revised and Enlarged Sasang Constitutional Medicine, Jipmoondang, Seoul, South Korea, 2012.

[21] R. Z. Zhang, S. J. Yu, H. Bai, and K. Ning, "TCM-mesh: the database and analytical system for network pharmacology analysis for TCM preparations," Scientific Reports, vol. 7, no. 1, pp. 1-14, 2017.

[22] D. Szklarczyk, A. Santos, C. von Mering, L. J. Jensen, P. Bork, and M. Kuhn, "STITCH 5: augmenting protein-chemical interaction networks with tissue and affinity data," Nucleic Acids Research, vol. 44, no. D1, pp. D380-D384, 2016.

[23] H. Heberle, G. V. Meirelles, F. R. da Silva, G. P. Telles, and R. Minghim, "InteractiVenn: a web-based tool for the analysis of sets through Venn diagrams," BMC Bioinformatics, vol. 16, pp. 1-7, Article ID 169, 2015.

[24] H. Mi, A. Muruganujan, X. Huang et al., "Protocol update for large-scale genome and gene function analysis with the PANTHER classification system (v.14.0)," Nature Protocols, vol. 14, no. 3, pp. 703-721, 2019.

[25] X. Fu, L. H. Mervin, X. Li et al., "Toward understanding the cold, hot, and neutral nature of Chinese medicines using in silico mode-of-action analysis," Journal of Chemical Information and Modeling, vol. 57, no. 3, pp. 468-483, 2017.
[26] A. Gutiérrez-Sacristán, J. Deu-Pons, E. Centeno, J. GarcíaGarcía, F. Sanz, and L. I. Furlong, "DisGeNET: a comprehensive platform integrating information on human diseaseassociated genes and variants," Nucleic Acids Research, vol. 45, no. D1, pp. D833-D839, 2017.

[27] World Health Organization, International Statistical Classification of Diseases and Related Health Problems, 10th Revision (ICD-10), World Health Organization, Geneva, Switzerland, 2016.

[28] D. Parisi, M. F. Adasme, A. Sveshnikova, S. N. Bolz, Y. Moreau, and M. Schroeder, "Drug repositioning or target repositioning: a structural perspective of drug-target-indication relationship for available repurposed drugs," Computational and Structural Biotechnology Journal, vol. 18, 2020.

[29] J. P. Després, "Lipoprotein metabolism in visceral obesity," International Journal of Obesity, vol. 15, pp. 45-52, 1991.

[30] B. V. Howard, "Insulin resistance and lipid metabolism," The American Journal of Cardiology, vol. 84, no. 1, pp. 28-32, 1999.

[31] C.-H. Lee, P. Olson, and R. M. Evans, "Minireview: lipid metabolism, metabolic diseases, and peroxisome proliferatoractivated receptors," Endocrinology, vol. 144, no. 6, pp. 2201-2207, 2003.

[32] T.-G. Lee, B. Koh, and S. Lee, "Sasang constitution as a risk factor for diabetes mellitus: a cross-sectional study," EvidenceBased Complementary and Alternative Medicine, vol. 6, no. s1, 5 pages, Article ID 425165, 2009.

[33] E. Jang, Y. Baek, K. Park, and S. Lee, "The sasang constitution as an independent risk factor for metabolic syndrome: propensity matching analysis," Evidence-Based Complementary and Alternative Medicine, vol. 2013, Article ID 492941, 6 pages, 2013.

[34] S. Zhu, L. Min, Q. Guo et al., "Transcriptome and methylome profiling in a rat model of irritable bowel syndrome induced by stress," International Journal of Molecular Medicine, vol. 42, 2018.

[35] E. S. Jang, H. S. Kim, J. H. Yoo, S. H. Kim, Y. H. Baek, and S. W. Li, "The verification of concordance coefficient in sasang constitutional factors by expert," Journal of Sasang Constitutional Medicine, vol. 21, no. 2, pp. 79-86, 2009.

[36] H. J. Jin, Y. Baek, H. S. Kim, J. Ryu, and S. Lee, "Constitutional multicenter bank linked to Sasang constitutional phenotypic data," BMC Complementary and Alternative Medicine, vol. 15, 2015. 